\title{
The Anisotropy of Cosmic Rays and the Global Anisotropy of Physical Space
}

\author{
Yuriy Alexeevich Baurov \\ Closed Joint Stock Company Research Institute of Cosmic Physics, Moscow, Russia \\ Email: baurov@mail.ru
}

Received September 7, 2012; revised October 6, 2012; accepted October 13, 2012

\begin{abstract}
The influence of a new anisotropic factor onto the mechanism of accelerating cosmic rays up to ultrahigh energies (CR UHE) due to a new global natural force with the anisotropic behavior is considered. The directions in the physical space along which CR UHE can arrive, are predicted. A brief comparative analysis of these directions together with the obtained experimental results is given. Their qualitative coincidences are shown.
\end{abstract}

Keywords: Anisotropy of Cosmic Rays; Global Anisotropy; New Force

\section{Introduction}

In recent years much attention is given to the research of cosmic rays with ultrahigh energies (CR UHE) in the region of Greisen-Zatsepin-Kuzmin energy limit (GZK) $5 \times 10^{19} \mathrm{eV}$ as well as to the investigation of the anisotropy of directions of cosmic particles arrival to the Earth [1-3]. The direct measurements give only some data for the directions of $\gamma$-quanta arrival straight from the sources. The charged particles of cosmic rays undergo the scattering in the magnetic fields of the Galaxy so that the directions to the sources can be determined only statisticcally by way of observing the anisotropy of particles arrival (i.e. the excess of the number of arriving particles along the direction to the source over the expected number in case of isotropy). With increase in energy of particles the scattering in the magnetic fields decreases, and the anisotropy in directions of their arrival is built up. Therefore the special attention of researchers is drawn by the studying the cosmic rays of ultrahigh energy above the GZK-limit $\left(\sim 10^{20} \mathrm{eV}=100 \mathrm{EeV}\right)$ for which the deflections in the galactic fields are so small that we can hope for the direct observation of the source on the celestial sphere. To observe a sufficiently great number of particles of such high energies, the installations with enormous operation areas (thousands $\mathrm{km}$ ) are necessary. At present there is built and gives results the most large ground facility "The Pierre Auger's observatory" in the South Hemisphere (Argentina). In the North hemisphere (USA) the ground "Telescopic facility" is began to function. The both ground facilities allow for studying the directions of coming of CR UVE particles over the whole celestial sphere. But the global investigation of CR UVE with the aid of ground facilities meets with the procedural difficulties associated with the different estimations of energies of primary particles in each detector which leads, due to fast fall of cosmic particles intensity with the energy, to large differences in experimental energy estimations for these particles. In this connection the creation of space detectors of cosmic rays is gaining in importance. Such detectors are preparing presently for the MSU satellite "Mikhailo Lomonosov" under the RF's Federal Cosmic Program with the participation of scientists from Korea, Mexico, Japan [4], and for the International Space Station (the detector JEM-EUSO) under the program of Japan Cosmic Agency with collaboration of scientists from many countries including RF [5]. The advantage of a space detector is its important ability to scan the whole celestial sphere and to obtain the intensity chart of coming CR UHE particles without corrections for distinctions in the energy estimations made for various parts of the celestial sphere. This allows for the more evident detecting the possible anisotropy of cosmic rays that is actively discussed in the literature [1-3,6-10].

In connection with the advancement in the experimental investigation of CR UHE, much attention is given to consideration of theoretic models of their origin [11].

The topic of the present paper is the estimation of possible connection between, on the one part, the anisotropy in directions of CR particles arrival and, on the other part, the assumed global anisotropy of the physical space and the associated new physical interaction [12-26] as the evidences from one of such "nonstandard" models $[16,17,23]$. 


\section{The "Standard" Model of CR UHE Origin}

Before to go on to the basic item of the paper, let us give a brief revue of the "standard" theory of CR UHE origin. The basic model is that of the acceleration of charged particles (protons and nuclei with various atomic numbers) at the front of a high speed gas "shock wave". This model is characterized by the maximum attainable energies of accelerated particles: on the order of $E_{\max } \sim(u / c)$ $Z B L$ (in EeV units) where $u$ is the gas velocity, $c$ is the light speed, $Z$ the charge of particle, $B$ the magnetic induction (in microgausses), and $L$ is the dimension of the acceleration region (in kiloparsecs). For the galactic cosmic rays, the accelerators are represented by the Supernovas envelopes for which $u / c \sim 0.1, B \sim 1, L \sim 0.1$ so that, in accordance with experimental data, $E_{\max } \sim 0.01$ $\mathrm{EeV}$ for the protons $(Z=1)$ and $E_{\max } \sim 1 \mathrm{EeV}$ for the iron nuclei. The search for the astrophysical objects capable of accelerating particles up to $10-100 \mathrm{EeV}$ leads to the objects that contain gaseous jets moving with high speeds $(u / c \sim 1)$ in strong magnetic fields $B$ and have large dimensions $L$. Those are cores of active galaxies $(B$ $\left.\sim 10^{6}, L \sim 1\right)$, radio-galaxies $\left(B \sim 10^{4}, L \sim 100\right)$, and colliding galaxies $\left(B \sim 2 \times 10^{4}, L \sim 30\right)$.

\section{The New Physical Models and the Origin of CR UHE}

In the "nonstandard" models, the particles with the energy on the order of $\sim 100 \mathrm{EeV}$ can be products of decay of hypothetic heavy particles (mass on the order of $\sim 10^{24}$ $\mathrm{eV}$ ) being the remainders of the Bing Bang [11]. Such particles may be responsible for the dark matter, in particular, for that of the Milky Way. In such a case an asymmetry in the arrival of CR UHE particles relative to the center of our Galaxy must be observed since the Solar system together with the Earth is at the periphery of the Galaxy. The modern experimental data on the directions of arrival of particles with the energy of $10-100$ $\mathrm{EeV}$ are in contrast with this hypothesis.

Another "nonstandard" model is that founded on the new type of interaction between the natural objects. The evidences for the existence of such interaction are obtained in many ground experiments and astrophysical observations.

In the papers [12-14] the results of experiments are described where this non-gauge interaction different from the four known ones (strong, weak, electromagnetic, and gravitational) was firstly detected. Besides the lack of gauge invariance, an important characteristic feature of this new interaction is its anisotropy that manifests itself in a wide range of dimensions: from those for weak interactions $\left(10^{-17} \mathrm{~cm}\right)[16-21]$ up to the size of our Galaxy $\left(10^{22} \mathrm{~cm}\right)$ and more $[16,17,22,27]$. The magnitude of the force measured was on the level of $0.01-0.08 \mathrm{gf}$ at the magnetic field of $10-13 \mathrm{~T}$ in the $40-53 \mathrm{~mm}$ solenoid aperture and at plumb mass of $26-30 \mathrm{~g}$.

The physical nature of the new force becomes clear from the byuon theory (the non-gauge theory of formation of the physical space and the world of elementary particles from some unobservable objects "byuons") $[16$, $17,23]$. It follows from that theory that there is a possibility of acting upon the process of mass formation of the elementary particles by the potentials of physical fields since a part of mass of particles associated with the formation of their inner space, is proportional to the modulus of a summary potential $\mathbf{A}_{\Sigma}$ that cannot exceed, by magnitude, the modulus of the cosmological vectorial potential $\mathbf{A}_{\mathbf{g}}$, a new fundamental constant introduced in [17-19] $\left(\left|\mathbf{A}_{\mathbf{g}}\right|=1.9 \times 10^{11} \mathrm{Gs} \mathrm{cm}\right)$. As the result of the action of the field potentials (decrease in $\left|\mathbf{A}_{\Sigma}\right|$ ), each particle gains an energy $\Delta \mathrm{mc}^{2}$ that corresponds to a new natural force throwing substance out of the region with the weakened $\mathbf{A}_{\boldsymbol{\Sigma}}$.

The further experimental investigations of the assumed new interaction with the use of gravimeters with attached magnet [15-17] and plasma systems $[16,17,24,25]$, as well as the measurements of changes in the $\beta$-decay rate of radioactive elements [16-21] have shown that the substance is ejected from the region with the weakened $\mathbf{A}_{\boldsymbol{\Sigma}}$ along a cone with an opening of $\sim 100^{\circ}$ around the vector $\mathbf{A}_{\mathbf{g}}$ determining the global anisotropy of the physical space and having the following coordinates in the second equatorial system: $\alpha \approx 293^{\circ} \pm 10^{\circ}, \delta \approx 36^{\circ} \pm 10^{\circ}$, ( $\alpha$ : right ascension, $\delta$ : declination). The revealed anisotropic properties of the physical space are confirmed by numerous astrophysical observations: the anisotropy in the motion of pulsars in the picture plane [17,22,27], the distribution of earthquakes relative the stars $[17,26]$ etc.

As the new force acts on all particles having mass, the manifestations of the new natural force's anisotropic properties can be the cause of the observed anisotropy of the cosmic rays. We will try that in the present paper but it should be noted that the new force has isotropic properties, too. In [23] the idea of the new natural force is used to explain such known astrophysical notion as the dark energy. It is shown that at distances on the order of $10^{27}-10^{28} \mathrm{~cm}$ the new force begins to exceed the gravitation and diminishes $\mathbf{A}_{\Sigma}$ pushing galaxies aside due to the negative value of the gravitational potential.

\section{The Experimental Data on the Anisotropy of Cosmic Rays and the Acceleration of Particles by the New Global Force}

Figure 1 gives the results of an experiment on investigating the anisotropy of cosmic rays with the energy $\sim 10$ $\mathrm{TeV}$ using the detectors of Milagro, Tibet, and $\mathrm{Su}-$ per-Kamiokande [1-3]. As is seen from the Figure, there 


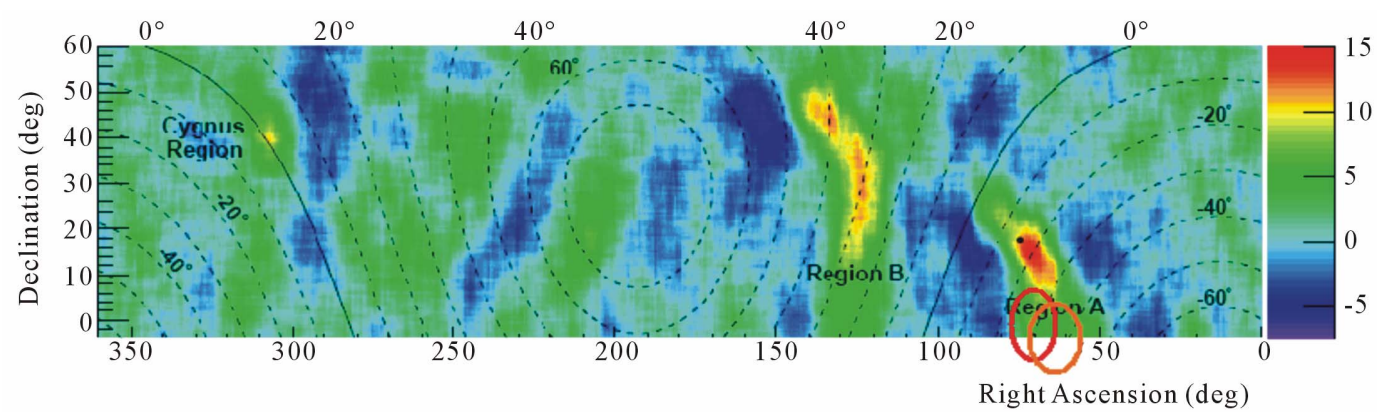

Figure 1. The directions of arrival of cosmic ray particles with the energy of $1-10 \mathrm{TeV}$ according to data from the detectors: Milagro (basic map), Tibet (red ellipse), and Super-Kamiokande (pink ellipse). The increase of intensity: dark blue $\rightarrow$ red.

exist several regions with predominant arrival of particles. These regions have the following coordinates in the second equatorial system: Region $\mathrm{A}\left(\alpha=70^{\circ} \pm 10^{\circ}, \delta=0^{\circ} \pm\right.$ $\left.20^{\circ}\right)$, region $\mathrm{B}\left(\alpha=130^{\circ} \pm 15^{\circ}, \delta=30^{\circ} \pm 20^{\circ}\right)$, Cygnus Region $\left(\alpha=305^{\circ} \pm 5^{\circ}, \delta=40^{\circ} \pm 5^{\circ}\right)$. The detected anisotropy of cosmic rays is not large, on the level of $10^{-3}$ from the isotropic flux of particles. The reason is that the particles with energies of the $10 \mathrm{TeV}$ order in large part loose the initial direction due to the scattering in the galactic magnetic fields. The experimenters seek explanation of the anisotropy detected in an additional acceleration of particles at the front of collision of the helioplasma with the interstellar medium [1-3].

At the same time that anisotropy may be possibly explained by the additional acceleration of particles by the new global anisotropic force.

The new force is nonlinear and can be represented in the form of some series in the change of $\Delta A_{\Sigma}$. The first term of the series is

$$
F=2 N m_{v} c^{2} \lambda_{1}^{2} \Delta A_{\Sigma}\left(\Delta A_{\Sigma} / \Delta X\right),
$$

where $N$ is the number of stable particles in the body considered (the number of electrons, protons, and neutrons), $2 m_{1} c^{2} \approx 33 \mathrm{eV}$ is the magnitude of the proper energy of particles that can be influenced, with the probability 1 , by the potentials of fields, $\lambda_{1}$ the first dimensional series factor equal to $10^{-12}(\mathrm{Gs} \mathrm{cm})^{-1}$, and $\Delta X$ is the change in the space coordinate.

Since the proton has a magnetic moment [28] there is reason to assume that the new force may be, in the cosmic space, a potent accelerator of protons and other charged particles having magnetic moment and, consequently, a considerable magnitude of $\Delta A_{\Sigma} / \Delta X$. For the proton $\Delta A_{\Sigma} / \Delta X=10^{16}$ Gs [19]. After substituting into Equation (1) and assuming $N=1, \Delta A_{\Sigma}=10^{11} \mathrm{Gs} \mathrm{cm}$ we have $F \approx 10^{-7}$ dyne.

During the motion in the Universe the proton can cover, in the vicinity of galaxies, space distances where $\Delta A_{\Sigma} \approx 10^{11} \mathrm{Gs} \mathrm{cm}$. This value is the limiting one for our Universe and can be caused by the action on $\Delta A_{\Sigma}$ as by vector potentials of magnetic fields so by gravitational potentials [23].

It is commonly supposed that the protons in the Galaxy diffuse to the Earth for ten thousand years so that the whole distance $L \approx 10^{22} \mathrm{~cm}$ (the measure of a medium-sized galaxy is $\sim 10^{24} \mathrm{~cm}$ [29]). If the force considered acts on scales of $L$, the energy imparted to the proton by the new force will be of order $10^{27} \mathrm{eV}$ (much more then the GZK limit). It should be noted that to estimate the energy of cosmic rays we have used the linear approximation (1) that gives, as the ground experiments show $[12-14,16,17]$, nearly an order of magnitude greater value for the new force of nature. In addition, the interaction with the relic radiation will naturally diminish the value of energy obtained but the anisotropic properties of the assumed new force are bound to affect the anisotropy of CR UHE. The coordinates of their arrival to the Earth will be in correspondence with the opposite directions to the new force cone of acting. Figure 2 demonstrates, in the plane of the ecliptic, the directions of action of the new force, of the $\mathbf{A}_{\mathbf{g}}$ vector, of vector-potentials from Earth's and Sun's magnetic fields, as well as the supposed directions of CR UHE arrival for the characteristic data of motion of the Earth around the Sun (March 21 and December 21). As an example, there are also shown results of an investigation into the anisotropy of CR with the energy of $10 \mathrm{TeV}$ that were measured with the use of the Super-Kamiokande detector [1]. As is seen from the Figure, the basic direction of the anisotropy found in this experiment $\left(\alpha=75^{\circ} \pm 7^{\circ}, \delta=-5^{\circ} \pm 7^{\circ}\right)$ is in correspondence with one of the new force directions, and the found cone of CRs spread $\left(75^{\circ} \pm 7^{\circ}\right)$ practically overlays the predicted approach directions of cosmic rays.

It should be noted that the anisotropy found with the aid of the Super-Kamiokande 1 detector is not great: the significance level of the result is no more than $13 \%$. This can be explained by the fact that the cosmic rays Registered by this detector fall in the $\mathrm{CR}$ of galactic origin instead of CR UHE. Nevertheless, the arrival of CR from the regions $\mathrm{B}\left(\alpha=130^{\circ} \pm 15^{\circ}, \delta=30^{\circ} \pm 20^{\circ}\right.$, see Figure 1) is in the correspondence with another direction predicted in Figure 2. The Cygnus Region $\left(\alpha=305^{\circ} \pm 5^{\circ}, \delta=40^{\circ}\right.$ 


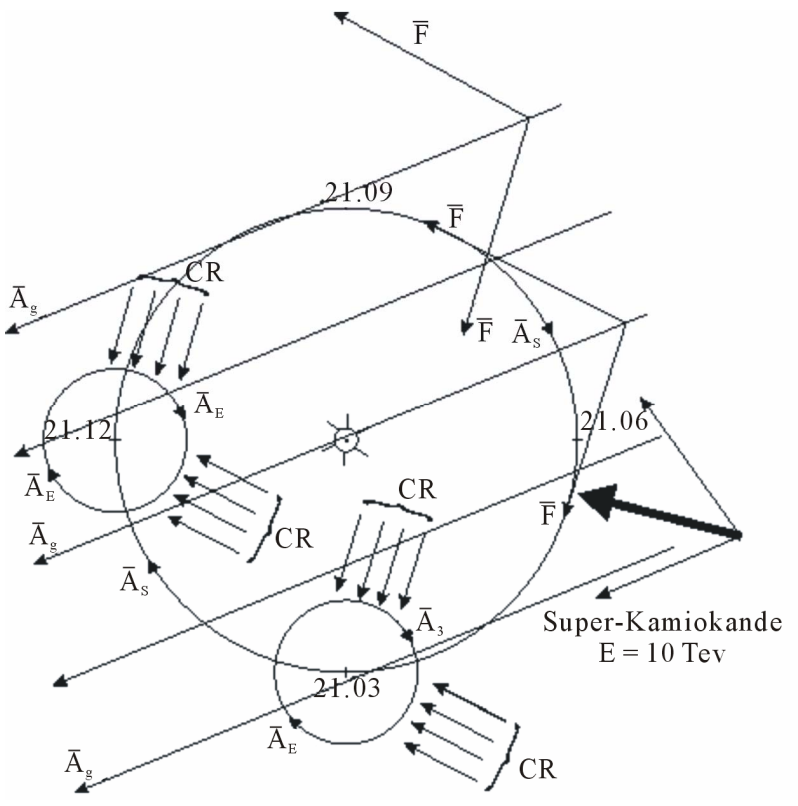

Figure 2. The predicted directions of arrival of CR UHE accelerated by the new force of nature $F$. $A_{g}$ - the direction of the cosmological vector potential; $A_{E}$ - the direction of the vector potential of the Earth's magnetic field; $A_{S}$ - the direction of the vector potential of the Sun's magnetic field (dipolar with 2000 year); 21.03 - the characteristic data of the Earth's orbiting around the Sun; F-the directions of the new force action; CR-the predicted directions of CR UHE arrival in the characteristic days 21.03 and 21/12.

$\pm 5^{\circ}$ ) shown in Figure 1 practically corresponds to the direction of the cosmological vector potential $\mathbf{A}_{\mathbf{g}}$ (see above). The cause can be that the $\mathrm{CR}$ accelerated by the new force can, when interacting with substance, give rise to $\gamma$-quanta that move precisely in the opposite direction to the global anisotropy associated with the existence of vector $\mathbf{A}_{\mathbf{g}}$.

With increasing energy of particles observed the anisotropy is bound to increase, too. But to determine the primary arrival direction along the new force for the particles with the most large energy $\left(>5 \times 10^{19} \mathrm{eV}\right)$ that are weakly scattered in the magnetic fields (lesser than $5^{\circ}$ for protons), there are presently short of data (Figure 2).

When studying cosmic rays in the region of spectrum break according to GZK (due to absorbing by relic photons), an important contribution can be made from extragalactic sources at a range lesser $100 \mathrm{Mps}$ from the Earth for which energy losses at collisions with the photons are small. When observing arrival directions of particles from these galaxies, the direction of the global force will influence along with the position of galaxy on the celestial sphere.

The role of the new force will be large if the acceleration of particles (for example, protons) due to this force is comparable with the acceleration thanks to the "standard" accelerating mechanism at the shock wave. The magnitude of force $F$ for accelerating a proton up to the energy on the order of $10^{20} \mathrm{eV}$ within the distance $L \mathrm{~cm}$ can be estimated from the relation $F L \times 6 \times 10^{11} \mathrm{eV}=$ $10^{20} \mathrm{eV}$. For a source with the dimension $L=10^{23} \mathrm{~cm}$ (radio-galaxy) the force $F$ is bound to be no lesser than $10^{-15}$ dyne. As was shown above, the magnitude of the new force can be some orders greater.

\section{Conclusion}

The results of experimental investigations of the cosmic rays anisotropy at considerably low energies $(\sim 10 \mathrm{TeV})$ and at the energies on the level of $1 \mathrm{EeV}$ denote the possible action of a new anisotropic force. To confirm these results, there are necessary further experiments on measuring the directions of arrival of cosmic rays of more high energy. The most quick CR particles $\left(>5 \times 10^{19} \mathrm{eV}\right)$ that weakly scatter in the magnetic fields of the Galaxy, are of special interest. At that the new anisotropic interaction is evidence of the presence of natural CR with energies much greater than the GZK limit.

\section{Ackonwledgements}

The author is grateful to B. A. Khrenov and M. Zotov, the researchers from the Research Institute of Nuclear Physics of Moscow University, for useful discussions and some materials on the investigation of CR UHE, as well as to E. P. Morozov for the translation of the paper into English.

\section{REFERENCES}

[1] G. Guillian, et al., "Observation of Anisotropy of $10 \mathrm{TeV}$ Primary Cosmic Ray Nulei Flux with the Super-Kamiokande-1 Detector," Physical Review D, Vol. 75, No. 6, 2007, Article ID: 062003. doi:10.1103/PhysRevD.75.062003

[2] P. A. Collab, "Update on the Correlation of the Highest Energy Cosmic Rays with Nearby Extragalactic Matter," Astroparticle Physics, Vol. 34, No. 5, 2010, pp. 314-326. doi:10.1016/j.astropartphys.2010.08.010

[3] A. V. Glushkov, "Multipolar Anisotropy of Cosmic Ray with $\mathrm{E} \geq 10^{17} \mathrm{eV}$ by Data of Ykutskoy Installation SHAL," Physics of Atomic Nuclei, Vol. 74, No. 1, 2011, pp. 60-67.

[4] V. Abrashkin, et al., "Updated TUS Space Fluorescence Detector for Study of UHECR," Advances in Space Research, Vol. 41, No. 12, 2008, pp. 2079-2088. doi:10.1016/i.asr.2007.03.036

[5] Y. Takahashi and JEM-EUSO Collaboration, "The JEMEUSO Mission," New Journal of Physics, Vol. 11, 2009, Article ID: 065009. doi:10.1088/1367-2630/11/6/065009

[6] E. Waxman, K. B. Fisher and T. Piran, "The Signature of a Correlation between $>10^{19} \mathrm{eV}$ Cosmic Ray Sources and Large Scale Structure," The Astrophysical Journal, Vol. 483, No. 1, 1997, pp. 1-7. 
[7] N. W Evans, F. Ferrer and S. Sarkar, "The Anisotropy of the Ultra-High Energy Cosmic Rays," Astroparticle Physics, Vol. 17, No. 3, 2002, pp. 319-340.

[8] A. Cuoco, et al., "The Footprint of Large Scale Cosmic Structure on the Ultra-High Energy Cosmic Ray Distribution," Journal of Cosmology and Astroparticle Physics, Vol. 1, 2006, p. 9.

[9] O. E. Kalashev, et al. "Global Anisotropy of Arrival Directions of Ultra-High Energy Cosmic Rays: Capability of Space Based Detectors," Journal of Cosmology and Astroparticle Physics, Vol. 2008, No. 3, 2008, p. 5.

[10] A. Smialkowski, M. Giller and W. Michalak, "Luminous Infrared Galaxies as Possible Sources of the UHE Cosmic Rays," Journal of Physics G: Nuclear and Particle Physics, Vol. 28, No. 6, 2002, pp. 1359-1374.

[11] V. A. Kuzmin, I. I. Tkachev, "Matter Creation via Vacuum Fluctuations in the Early Universe and Observed Ultrahigh Energy Cosmic Ray Events," Physical Review D, Vol. 59, No. 12, 1999, Article ID: 123006. doi:10.1103/PhysRevD.59.123006

[12] Y. A. Baurov, E. Y. Klimenko and S. I. Novikov, "Experimental Observation of Space Magnetic Anisotropy," Doklady Akademii Nauk (DAN) USSR, Vol. 315,1990, pp. 1116-1120.

[13] Y. A. Baurov, E. Y. Klimenko and S. I. Novikov, "Experimental Observation of Space Magnetic Anisotropy," Physics Letters A, Vol. 162, No. 1, 1992, pp. 32-34. doi:10.1016/0375-9601(92)90954-K

[14] Y. A. Baurov, "Space Magnetic Anisotropy and a New Interaction in Nature," Physics Letters A, Vol. 181, No. 4, 1993, pp. 283-288. doi:10.1016/0375-9601(93)90609-4

[15] Y. A. Baurov and A. V. Kopaev, "Experimental Investigation of Signals of a New Nature with the Aid of Two High Precision Stationary Quartz Gravimeters," Hadronic Journal, Vol. 25, No., 2002, pp. 697-711.

[16] Y. A. Baurov, "On the Structure of Physical Vacuum and a New Interaction in Nature (Theory, Experiment and Applications)," Nova Science, New York, 2000.

[17] Y. A. Baurov, "Global Anisotropy of Physical Space, Experimental and Theoretical Basis," Nova Science, New York, 2004.

[18] Y. A. Baurov and V. L. Shutov, "About Influence of Vectorial Magnetic Potential of Sun and Earth in $\beta$-Decay
Rate," Applied Physics, Vol. 1, 1995, pp. 40-45.

[19] Y. A. Baurov, et al., "Experimental Investigations of Changes in $\beta$-Decay Rate of ${ }^{60} \mathrm{Co}$ and ${ }^{137} \mathrm{Cs}$," Modern Physics Letters A, Vol. 16, No. 32, 2001, pp. 2089-2101. doi:10.1142/S0217732301005187

[20] Y. A. Baurov, et al., "Experimental Investigation of Changes in $\beta$-Decay Rate of the Radioactive Elements," Physics of Atomic Nuclei, Vol. 70, No. 11, 2007, pp. 1825-1835. doi:10.1134/S1063778807110014

[21] Y. A. Baurov, et al., "Preliminary Results of ${ }^{60} \mathrm{Co}$ $\beta$-Decay Rate Change Long-Term Experimental Investigation in 2010", Applied Physics, Vol. 5, 2011, pp. 12-21.

[22] Y. A. Baurov, A. A. Shpitalnaya and I. F. Malov, "Global Anisotropy of Physical Space and Velocities of Pulsars," International Journal Pure and Applied Physics, Vol. 1, No. 1, 2005, pp. 71-82.

[23] Y. A. Baurov and I. F. Malov, "On the Nature of Dark Matter and Dark Energy," Journal Modern Physics, Vol. 1, No. 1, 2010, pp. 17-32. doi:10.4236/jmp.2010.11003

[24] Y. A. Baurov, et al., "Experimental Investigation of the Distribution of Pulsed-Plasma-Generator at Its Various Spatial Orientation and Global Anisotropy of Space," Physics Letters A, Vol. 311, No. 6, 2003, pp. 512-523. doi:10.1016/S0375-9601(03)00567-X

[25] Yu. A. Baurov, V. G. Farafonov and A. G. Znak, "Experimental Investigation of Heat Content in the Jet of Magnetoplasmadynamic Accelerator in Accordance with its Spatial Orientation", In: F. Gerard, Eds., Advances in Plasma Physics, Nova Science, New York, 2007, pp. 179-196.

[26] Y. A. Baurov, et al., "Seismic Activity of the Earth, the Cosmological Vectorial Potential and Method of a ShortTerm Earthquakes Forecasting," Natural Science, Vol. 3, No. 2, 2011, pp. 109-119. doi:10.4236/ns.2011.32016

[27] I. F. Malov and Y. A. Baurov, "The Distribution of the Spatial Velocities of Radio Pulsars," Astronomy Reports, Vol. 51, No. 10, 2007, pp. 830-835. doi:10.1134/S1063772907100071

[28] Yu. M. Shirokov and N. P. Yudin, "Nuclear Physics," Nauka, Moscow, 1980.

[29] “Astronomy: Century XXI,” In: V. G. Surdin, Ed., Fryazino: Century XX, 2007. 